\title{
Comparison of different methods of inter-eye asymmetry of rim area and disc area analysis
}

${ }^{1}$ Department of Ophthalmology, Centre de Recherche de I'Hôpital Maisonneuve-Rosemont Université de Montréal, Montreal, Quebec, Canada

${ }^{2}$ Department of Statistics, Université de Montréal, Montreal, Quebec, Canada

${ }^{3}$ Montreal Glaucoma Institute, Montreal, Quebec, Canada

Correspondence:

PJ Harasymowycz, Department of

Ophthalmology, Maisonneuve Rosemont Hospital, 5415 boul. de l'Assomption, Montreal, QC H1T 2M4, Canada Tel: + 15142523400 (ext. 4959);

Fax: + 15142517094

E-mail: pavloh@

igmtl.com

Received: 2 September 2010

Accepted in revised form: 20 July 2011

Published online:

16 September 2011

Presented at Association for Research in Vision and Ophthalmology Annual Meeting, Fort Lauderdale, FL, USA, May 2006.
Abstract

Purpose To describe different methods of inter-eye asymmetry of rim area (RA) to disc area (DA) asymmetry ratio (RADAAR) analysis.

Methods This was an observational, descriptive, and cross-sectional study. Both the eyes of all participants underwent confocal scanning laser ophthalmoscopy (Heidelberg retina tomograph (HRT 3)), frequencydoubling technology perimetry (FDT), and complete ophthalmological examination. Based on ophthalmological clinical examination and FDT results of the worse eye, subjects were classified as either normal, possible glaucoma, and probable glaucoma or definitive glaucoma. RADAAR values were calculated based on stereometric HRT 3 values using different mathematical formulae.

RADAAR-1 was calculated as a relative difference of rim and DAs between the eyes. RADAAR-2 was calculated by subtracting the value of rim to DA ratio of the smaller disc from the value of rim to DA ratio of the larger disc. RADAAR-3 was calculated by dividing the previous two values. Statistical analyses included ANOVA as well as Student $t$-tests. Results Data of 334 participants were analysed, 78 of which were classified as definitive glaucoma. RADAAR-1 values were significantly different between the four different groups of diagnosis $(F=5.82$; $P<0.001)$. The 1st and 99th percentile limits of normality for RADAAR-1, RADAAR-2, and RADAAR-3 in normal group were, respectively, -10.64 and $8.4 ;-0.32$ and 0.22 ; and 0.58 and 1.32 .

Conclusions RADAAR-1 seems to best distinguish between the diagnostic groups. Knowledge of RADAAR distribution in various diagnostic groups may aid in clinical
AAK Fansi' ${ }^{1}, \mathrm{H}$ Boisjoly ${ }^{1}, \mathrm{M}$ Chagnon ${ }^{2}$

and PJ Harasymowycz ${ }^{1,3}$ diagnosis of asymmetric glaucomatous damage.

Eye (2011) 25, 1590-1597; doi:10.1038/eye.2011.217; published online 16 September 2011

Keywords: open-angle glaucoma; optic nerve head; asymmetry; HRT 3; RADAAR

Introduction

The early diagnosis of glaucoma remains a major issue in current ophthalmic research. Traditionally, it is based on the evaluation of optic discs and visual fields of subjects. Knowing that optic disc changes usually precede detectable visual field loss in early glaucoma, ${ }^{1-4}$ imaging devices, such as confocal scanning laser ophthalmoscope, are now used to evaluate optic discs.

The Heidelberg retina tomograph, (HRT, Heidelberg Engineering, Heidelberg, Germany), a type of a confocal scanning laser ophthalmoscope, produces a composite image of the optic nerve composed of 32 coronal sections, allowing a three-dimensional view of the optic nerve and quantification of various structural parameters. The neuroretinal rim asymmetry is an important factor in diagnosing glaucoma. And it has been found to be superior to the cup to disc ratio for glaucoma diagnosis. ${ }^{5,6}$ Another parameter has recently been described, namely rim area (RA) to disc area (DA) asymmetry ratio (RADAAR). This parameter was stated based on the fact that by comparing the two eyes of each subject, asymmetry takes into account inter-individual measures variation. Moreover, RADAAR seems to describe normality with consistency and it correlates significantly with intraocular pressure (IOP) and degree of the severity of glaucomatous optic nerve damage in patients 
with glaucoma. ${ }^{7,8}$ Harasymowycz et $a l^{7}$ and Hawker et $a l^{8,9}$ used different formulae to express inter-eye asymmetry. Given that the choice of the method for calculating asymmetry is trivial and that there is no consensus yet, the present study aims to describe and compare the inter-eye asymmetry of RA and DA (RADAAR) using different formulae in different diagnostic groups.

\section{Materials and methods}

\section{Study population}

This was an observational, descriptive, and cross-sectional study. Study subjects were recruited from a tertiary care glaucoma clinic at Maisonneuve-Rosemont Hospital as well as from mobile glaucoma-screening clinic sessions (GSCS) organised at differents centres in Montreal, Canada, during a 5-year period (July 2003-July 2008). The study was approved by the ethics board of Maisonneuve-Rosemont Hospital; written, informed consent was obtained from all subjects and the study was conducted in accordance with the Declaration of Helsinki. Inclusion criteria for subjects from the GSCS have been previously published. ${ }^{10}$ The present study includes only subjects older than 50 years of age. The exclusion criterion was inability to perform an HRT or an ophthalmological examination.

\section{Ophthalmological examination}

All subjects underwent a standard ophthalmological examination performed by the same glaucoma specialist (PJH). It included gonioscopy, IOP measurement, slit-lamp examination, and observation of the optic disc, nerve fibre layer, and retina after pupil dilation. Glaucomatous optic nerve damage was documented using vertical cup/disc ratio and the Disc Damage Likelihood Scale (DDLS), where stage 0 represents no damage and stage 7 far advanced rim loss. ${ }^{11}$ Based on the optic nerve and retinal nerve fibre layer ophthalmic examination results for each participant, individual eyes were classified as being normal (DDLS 0-1), glaucoma suspect (DDLS 2-3), or glaucoma (DDLS 4-7).

\section{Frequency-doubling technology}

All subjects had visual field testing in both the eyes using the frequency-doubling technology perimetry (FDT) screening C-20-5 programme (FDT, Carl Zeiss Meditec Inc., Dublin, CA, USA). For the C-20-5 algorithm, the test was explained to each subject, and a preview of the target stimuli was shown at the beginning. Both the eyes were tested according to the instrument protocol. Testing was performed in a dark room. In all, 17 targets were presented within the central 20 degrees of field. The FDT visual field had to be reliable (false positives less than $33 \%$, false negatives less than $33 \%$, and fixation losses less than 33\%). The screening results are displayed with four qualitative loss classifications ('within normal limits', 'mild relative loss', 'moderate relative loss', and 'severe loss') based on age-related normative references. Abnormal FDT included at least two adjacent points of mild relative loss in C-20-5 algorithm. ${ }^{12}$ Also visual field damage was documented using Patel et al ${ }^{13}$ score where score 0 represents no damage, and score 87 far advanced visual field loss. This score was derived from an interpretation algorithm where the values are assigned based on both the degrees or depth of defect and the location thereof. Thus, a value of 1 was assigned to the outside 12 points, a value of 3 to the inner four loci, and a value of 5 to fixation. Each point was graded from $0-3$ on the basis of the depth of defect. Normal areas were assigned a value of 0 , mild defects were graded as 1 , moderate as 2, and severe as 3 on the basis of the FDT gray-scale printout. This score of 0 through 3 was multiplied by the weighting factor of 1,3 , or 5 . A final score was determined by summing all abnormal points with ranging from 0 for a completely normal test to 87 for a test in which all points were missed at the maximum.

Normal, possible glaucoma, probable glaucoma, and definitive glaucoma subject classification based on the worse eye

The results of the ophthalmic examination and FDT were combined to classify eyes into four diagnostic categories (Table 1). Each eye of subjects that were normal based on ophthalmic examination with normal visual fields on

Table 1 Diagnostic classifications based on clinical examination and frequency-doubling technology (FDT) visual fields results

\begin{tabular}{lcl}
\hline Diagnostic groups & \multicolumn{2}{c}{ Examination results } \\
\cline { 2 - 3 } & Ophthalmic examination & FDT result \\
\hline Normal (A) & Normal & Normal \\
Possible glaucoma (B) & Normal & Abnormal \\
& Glaucoma suspect & Normal \\
Probable glaucoma (C) & Glaucoma suspect & Abnormal \\
& Glaucoma & Normal \\
Definitive glaucoma (D) & Glaucoma & Abnormal
\end{tabular}

Ophthalmic examination based on vertical cup/disc ratio and the Disc Damage Likelihood Scale (DDLS). ${ }^{11}$ DDLS classification: normal (DDLS 0-1), glaucoma suspect (DDLS 2-3), or glaucoma (DDLS 4-7). Abnormal FDT included at least two adjacent points of mild relative loss in C-20-5 algorithm. ${ }^{12}$ 
FDT was classified as 'normal'. Those who were normal on ophthalmic examination with abnormal visual fields on FDT or glaucoma suspect on ophthalmic examination with normal visual fields on FDT were classified as 'possible glaucoma'. Those who were glaucoma suspect on ophthalmic examination with abnormal visual fields on FDT or glaucoma on clinical examination with normal visual fields on FDT were classified as 'probable glaucoma'. Finally, subjects with a diagnosis of glaucoma on ophthalmic examination with abnormal visual field were classified as 'definitive glaucoma'. Only the eyes with good-quality HRT 3 exam and one good-quality FDT visual field in each eye within 6 months to the imaging date were included. Each subject was then classified as being normal, possible glaucoma, probable glaucoma, or definitive glaucoma based on the worse eye.

\section{Confocal scanning laser ophthalmoscopy}

All subjects also had a confocal scanning laser ophthalmoscopy (HRT 3 examination (HRT, version 3, Heidelberg Engineering) performed by the same trained ophthalmic photographer who outlined the optic disc margin. The working principle has been published. ${ }^{14,15}$ Image was in acceptable quality if the topography standard deviation (SD) value was inferior to 40 microns as defined by the manufacturer. Accommodation, distance of camera, blink and fixation loss, image brightness, eye movement, image illumination, and eye drift were also taken into account and the quality cheque had to be classified as least as 'good'. The HRT 3 examination results included Moorfields regression analysis (MRA) classification ${ }^{16}$ ('normal', 'borderline', and 'out of normal limits'), DA, cup area (CA), RA, and cup to DA ratio (CDAR).

\section{Asymmetry of variables}

RADAAR values were calculated based on global stereometric HRT 3 values using different formulae including Harasymowycz et $a l^{7}$ (RADAAR-1) and Hawker et $a l^{8,9}$ (RADAAR-2 and RADAAR-3).

RADAAR-1 was calculated as a relative difference of rim and DAs between the eyes (one minus RA of smaller DA divided by RA of larger DA; this all divided by one minus smaller DA divided by larger DA). RADAAR-2 was calculated by subtracting the value of rim to DA ratio of the smaller disc from the value of rim to DA ratio of the larger disc. RADAAR-3 was calculated by dividing the previous two values (Figure 1).

Asymmetry or inter-eye difference of other HRT 3 parameters including RA, CA, and CDAR were calculated by subtracting the value of the smaller disc
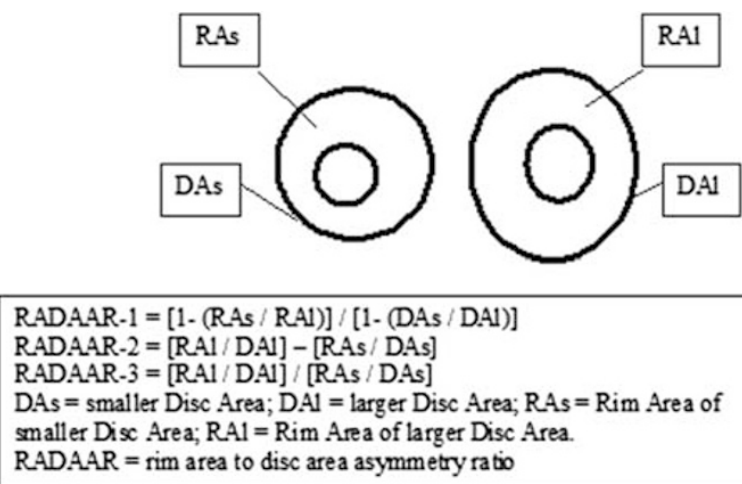

Figure 1 Different formulae used to illustrate inter-eye asymmetry.

from the value of a larger disc for each variable. Difference in DA was also calculated by subtracting the smaller DA from the larger DA. Differences for others variables including the IOP, the central corneal thickness (CCT), and the clinical-estimated DDLS were calculated in the same way.

\section{Statistical analysis}

Statistical analyses were performed using SPSS software version 16.0. (SPSS, Chicago, IL, USA). Statistical significance was set at $P<0.05$. ANOVA was used to compare the mean values of RADAAR using the different formulae for the four groups. Multiples comparisons were then made using the Tukey post-hoc test.

\section{Results}

\section{Study population characteristics}

Out of 523 subjects enrolled in the database available for this study, 77 subjects were from the tertiary care glaucoma clinic and 446 subjects were from mobile glaucoma-screening clinic session. A total of 95 subjects were excluded due to inability to have a good HRT examination. It included subjects in whom HRT exams had not been performed (74) or in whom HRT has been done in only one eye (9 subjects), and subjects with unreliable HRT based on criteria set forth in the Materials and methods section (12 subjects). Eight subjects also were excluded due to inability to have a FDT examination. Additionally 86 subjects were excluded due to unreliable FDT based on criteria state in the Materials and methods section, in both the eyes (22) in right eyes (23), and in left eyes (41) leaving 334 subjects for this study.

The diagnostic classification of subjects based both on clinical examination and visual fields of the worse eye included 108 normal, 121 possible glaucoma, 27 probable glaucoma, and 78 definitive glaucoma subjects. The 
characteristics of subjects from the four diagnostic groups are summarised in Table 2 . There were more females than males. The normal subjects were significantly younger than subjects of the other groups $(P<0.000)$. Inter-eye difference of most clinical parameters was statistically significantly different between the four groups of diagnosis (inter-eye IOP difference $(F=11.683 ; P<0.000)$, inter-eye DDLS difference $(F=13.19 ; P<0.000)$, and inter-eye clinical CDAR difference $(F=7.28 ; P<0.000))$. This statistical difference between the diagnostic groups was also found with clinical variables of worse eye of each subject (IOP of the worse eye $(F=73.03 ; P<0.00)$, DDLS of the worse eye $(F=37.9 ; P<0.000)$, and clinical CDAR of the worse eye $(F=25.47 ; P<0.000)$ ).

Table 3 displays the mean topography SD which indicates HRT 3 image quality and HRT 3 data for the study population, including DA, CA, RA, and CDAR. In all diagnostic groups, there was a statistically significant inter-eye difference between smaller and

Table 2 The study population characteristics

\begin{tabular}{|c|c|c|c|c|c|}
\hline & $\begin{array}{l}\text { Normal } \\
(A)\end{array}$ & $\begin{array}{c}\text { Possible glaucoma } \\
\text { (B) }\end{array}$ & $\begin{array}{l}\text { Probable glaucoma } \\
\text { (C) }\end{array}$ & $\begin{array}{l}\text { Definitive glaucoma } \\
\text { (D) }\end{array}$ & P-value \\
\hline Number of subjects & 108 & 121 & 27 & 78 & \\
\hline Mean age $\pm S D$ (years) & $58 \pm 9$ & $63 \pm 9$ & $68 \pm 11$ & $65 \pm 11$ & $<0.000^{*}$ \\
\hline Female/male $(\%)$ & $71 / 37$ & $88 / 33$ & $19 / 8$ & $42 / 36$ & \\
\hline Africans-Caribbeans/Caucasians (\%) & $17 / 91$ & $17 / 104$ & $4 / 23$ & $8 / 70$ & \\
\hline \multicolumn{6}{|l|}{$\mathrm{CCT}(\mathrm{OD} / \mathrm{OS})$} \\
\hline$n$ & $103 / 103$ & $118 / 118$ & $24 / 24$ & $73 / 73$ & \\
\hline Mean (SD) & $548(38) / 548(38)$ & $552(38) / 553(39)$ & $554(34) / 556(35)$ & $545(42) / 543(43)$ & \\
\hline \multicolumn{6}{|l|}{ Inter-eye CCT difference } \\
\hline Mean (SD) & $7.8(7.6)$ & $12.5(17.3)$ & $6.7(7.0)$ & $12.0(16.1)$ & $<0.034^{*}$ \\
\hline \multicolumn{6}{|l|}{$I O P(O D / O S)$} \\
\hline$n$ & 99/99 & $110 / 110$ & $25 / 25$ & $76 / 76$ & \\
\hline Mean (SD) & $16(3) / 16(3)$ & $16(3) / 16(3)$ & $17(5) / 18(5)$ & $27(10) / 28(11)$ & $<0.000^{*}$ \\
\hline \multicolumn{6}{|l|}{ Inter-eye IOP difference } \\
\hline Mean (SD) & $0.80(1.2)$ & $0.67(1.3)$ & $1.3(2.2)$ & $2.9(5.0)$ & $<0.000^{*}$ \\
\hline \multicolumn{6}{|l|}{ DDLS (OD/OS) } \\
\hline$n$ & $47 / 47$ & $79 / 79$ & $23 / 23$ & $36 / 36$ & \\
\hline Mean (SD) & 1.3(1.1)/1.3(1.3) & $1.5(1.2) / 1.9(1.4)$ & $3.0(1.1) / 2.8(1.1)$ & $4.3(1.5) / 4.7(1.3)$ & $<0.000^{*}$ \\
\hline \multicolumn{6}{|l|}{ Inter-eye DDLS difference } \\
\hline Mean (SD) & $0.30(0.5)$ & $0.48(0.8)$ & $0.96(1.02)$ & $1.5(1.6)$ & $<0.000^{*}$ \\
\hline \multicolumn{6}{|l|}{ Cup/disc ratio (OD/OS) } \\
\hline$n$ & $103 / 103$ & $112 / 112$ & $19 / 19$ & $10 / 10$ & \\
\hline Mean (SD) & $0.3(0.1) / 0.3(0.2)$ & $0.4(0.2) / 0.4(0.2)$ & $0.6(0.1) / 0.6(0.1)$ & $0.7(0.2) / 0.7(0.2)$ & $<0.000^{*}$ \\
\hline \multicolumn{6}{|l|}{ Inter-eye cup/disc ratio difference } \\
\hline Mean (SD) & $0.04(0.06)$ & $0.04(0.07)$ & $0.10(0.08)$ & $0.10(0.08)$ & $<0.000^{*}$ \\
\hline \multicolumn{6}{|l|}{ FDT score (OD/OS) } \\
\hline$n$ & $108 / 108$ & $121 / 121$ & $27 / 27$ & $78 / 78$ & \\
\hline Mean (SD) & $0.14(0.5) / 0.19(0.6)$ & $9.1(17.1) / 11.2(19.8)$ & $11.4(22.1) / 13.3(24.5)$ & $32.8(32.4) / 25.8(25.9)$ & $<0.000^{*}$ \\
\hline
\end{tabular}

Abbreviations: CCT, central corneal thickness; DDLS, Disc Damage Likelihood Score; FDT, frequency-doubling technology perimetry; IOP, intraocular pressure; $\mathrm{SD}$, standard deviation. $\mathrm{OD}$, right eye; $\mathrm{OS}$, left eye.

FDT score according to Patel et $a l^{13}$

The Tukey post-hoc test indicates that the statistically significant difference was between definitive glaucoma and normal subjects ((mean difference $= \pm 32.6, P<0.000$, for FDT score), (mean difference $= \pm 1.7, P<0.001$, for inter-eye IOP difference), (mean difference $= \pm 1.2, P<0.000$, for inter-eye DDLS difference)); between definitive glaucoma and possible glaucoma subjects ((mean difference $= \pm 23.7, P<0.000$ for FDT score), (mean difference $= \pm 1.7, P<0.001$, for inter-eye IOP difference), (mean difference $= \pm 1.0, P<0.000$, for inter-eye DDLS difference)); as well as between definitive glaucoma and probable glaucoma subjects ((mean difference $= \pm 1.5, P<0.001$, for inter-eye IOP difference), (mean difference $= \pm 0.6, P<0.017$, for inter-eye DDLS difference)).

${ }^{*} P$-value of ANOVA test, statistical significance level at 0.05 . 
Table 3 Heidelberg retina tomograph (HRT 3) measures for the study population

\begin{tabular}{|c|c|c|c|c|c|c|c|c|c|c|c|c|}
\hline & \multicolumn{3}{|c|}{$\begin{array}{l}\text { Normal mean } \\
\quad( \pm S D)\end{array}$} & \multicolumn{3}{|c|}{$\begin{array}{c}\text { Possible } \\
\text { glaucoma mean }( \pm S D)\end{array}$} & \multicolumn{3}{|c|}{$\begin{array}{c}\text { Probable } \\
\text { glaucoma mean }( \pm S D)\end{array}$} & \multicolumn{3}{|c|}{$\begin{array}{c}\text { Definitive } \\
\text { glaucoma mean }( \pm S D)\end{array}$} \\
\hline & $\begin{array}{c}\text { Smaller disc } \\
(\mathrm{N}=108)\end{array}$ & $\begin{array}{l}\text { Larger disc } \\
(\mathrm{N}=108)\end{array}$ & $\mathrm{P}$ & $\begin{array}{c}\text { Smaller disc } \\
(\mathrm{N}=121)\end{array}$ & $\begin{array}{l}\text { Larger disc } \\
(\mathrm{N}=121)\end{array}$ & $\mathrm{P}$ & $\begin{array}{l}\text { Smaller disc } \\
\qquad(\mathrm{N}=27)\end{array}$ & $\begin{array}{l}\text { Larger disc } \\
(\mathrm{N}=27)\end{array}$ & $\mathrm{P}$ & $\begin{array}{l}\text { Smaller disc } \\
\qquad(\mathrm{N}=78)\end{array}$ & $\begin{array}{l}\text { Larger disc } \\
(\mathrm{N}=78)\end{array}$ & $\mathrm{P}$ \\
\hline & $16.9(6.2)$ & 17.3 & & 6) & 21.0 & & 6) & 21.9 & & 21 & 22. & \\
\hline$\left(\mathrm{mm}^{2}\right)$ & $1.6(0.36)$ & 1.9 & 0.00 & 42) & 1.99 & 0.00 & 40) & 2.13 & 0.00 & 44) & 49) & 0.00 \\
\hline Cup area $\left(\mathrm{mm}^{2}\right)$ & $0.34(0.26)$ & $0.44(0.33)$ & 0.00 & $0.42(0.35)$ & $0.54(0.46)$ & 0.00 & $0.66(0.31)$ & $0.74(0.33)$ & 0.19 & $0.84(0.55)$ & $1.01(0.57)$ & 0.00 \\
\hline Rim area $\left(\mathrm{mm}^{2}\right)$ & $1.28(0.25)$ & $1.43(0.33)$ & 0.00 & $1.30(0.26)$ & $1.45(0.29)$ & 0.00 & $1.27(0.45)$ & $1.39(0.53)$ & 0.02 & $1.08(0.38)$ & $1.12(0.44)$ & 0.52 \\
\hline CDAR & $0.19(0.13)$ & $0.22(0.14)$ & 0.01 & $0.23(0.14)$ & $0.25(0.15)$ & 0.02 & $0.35(0.16)$ & $0.35(0.13)$ & 0.87 & $0.41(0.22)$ & $0.46(0.20)$ & 0.04 \\
\hline
\end{tabular}

Abbreviations: HRT 3, Heidelberg retina tomograph 3; SD, standard deviation.

HRT 3, confocal scanning laser ophthalmoscopy; SD HRT 3, mean topography standard deviation indicating HRT 3 image quality; CDAR, cup to disc area; Student $t$-tests $P$-value $=$ mean difference is significant at the 0.05 level.

larger value of HRT 3 parameters. RA mean value in the definitive glaucoma group, CA mean value, and CDAR mean value in the probable glaucoma group did not reach statistical significance. In addition, RA difference $(P<0.01)$, CA difference $(P<0.00)$, and CDAR difference $(P<0.00)$ were statistically different between groups with increasing difference from normal to glaucoma. DA difference was not statistically different between the groups.

\section{RADAAR formulae and the four different diagnostic groups}

The distribution of values for the RADAAR parameters using different formulae follows a bell-shaped distribution. Figure 2 shows the distribution of values of RADAAR-1 in normal, possible glaucoma, probable glaucoma, and definitive glaucoma groups illustrating a shift to the left and more variance in subjects with definitive glaucoma. The 1th and 99th percentile limits of normality for RADAAR-1, RADAAR-2, and RADAAR-3 were, respectively, -10.64 and $8.4 ;-0.32$ and 0.22 ; and 0.58 and 1.32 in the normal group. These results were, respectively, -14.34 and $15.50 ;-0.38$ and 0.18 ; and 0.52 and 1.3 in the possible glaucoma group. Also, these results were, respectively, -9.22 and $9.48 ;-0.38$ and 0.21 ; and 0.56 and 1.6 in the probable glaucoma group and finally, these results were, respectively, -95.68 and 16.35; -0.69 and 0.33 ; and 0.29 and 5.03 in the definitive glaucoma group.

The mean \pm SD of RADAAR values in normal, possible glaucoma, probable glaucoma, and definitive glaucoma groups were, respectively, $0.56 \pm 2.4,0.79 \pm 3.6,0.71 \pm 3.5$, and $-3.64 \pm 15.4$ for RADAAR-1. These results were, respectively, $-0.02 \pm 0.1,-0.02 \pm 0.09,-0.01 \pm 0.1$, and $-0.05 \pm 0.2$ for RADAAR-2 and finally these results were, respectively, $0.97 \pm 0.1,0.98 \pm 0.1,1.01 \pm 0.2$, and $1.01 \pm 0.6$ for RADAAR-3.

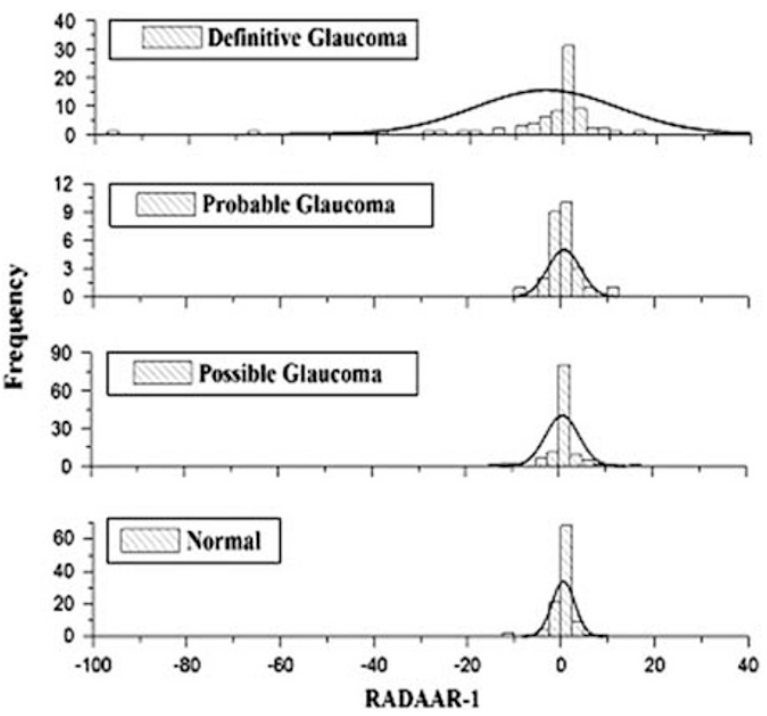

Figure 2 The distribution of RADAR-1 measures in normal, possible glaucoma, probable glaucoma, and definitive glaucoma groups.

There was a significant difference in central tendency (mean) of RADAAR-1 between the different diagnostic groups $(F=5.824, P<0.000)$. The Tukey post-hoc test indicates that this statistically significant difference was between definitive glaucoma and normal subjects (mean difference $= \pm 4.2, P<0.003$ ) as well as between definitive glaucoma and possible glaucoma subjects (mean difference $= \pm 4.42, P<0.001$ ).

\section{RADAAR formulae and clinical variables}

A correlation was found between RADAAR-1 and the IOP of the worse eye $(r=-0.128 ; . P<0.026)$ as well as the DDLS of the worse eye $(r=-0.164 ; P<0.028)$. Also, difference in inter-eye IOP significantly correlated with RADAAR-2 $(r=-0.168 ; P<0.003)$. A correlation was 
found between RADAAR-3 and the clinical CDAR of the worse eye $(r=-0.224 ; P<0.000)$.

\section{Discussion}

Although open-angle glaucoma may be bilateral and cause symmetric damage, a meticulous study of the discs will often reveal an asymmetry of the rim to disc ratio especially at an early stage of the disease. A study in an animal model showed that the progression of the disease was often asymmetric between the eyes of individual mice. ${ }^{17}$ The tendency towards asymmetrical development of congenital glaucoma was well illustrated by some authors showing unilateral development in about $25 \%$ of $^{\text {cases. }}{ }^{18}$

The role of asymmetry in early diagnosis of glaucoma is therefore indisputable and, in this context, three studies $^{7-9}$ have recently investigated the utility of asymmetry measures in glaucoma diagnosis using HRT.

As observed by Hawker et $a l^{8}$ asymmetry measures by subtracting the value of the right eye from that of the left eye may explain the insensitivity of the measure of asymmetry to detect glaucoma as found in The Blue Mountain Study, ${ }^{19}$ where only the vertical cup disc ratio was considered. Measuring the asymmetry between the small and large disc ensures a certain consistency of asymmetry that is less susceptible to changes with the optic disc and cup size. In addition to the potential of reducing the variability of HRT parameters by taking asymmetry into account along with age, ${ }^{20}$ gender, $^{21}$ $\mathrm{DA},{ }^{16,20,21}$ and refraction ${ }^{22}$ as found in previous studies, our study indicates that RADAAR may be useful to distinguish between normal and glaucoma patients. The current study describes distribution of three asymmetry formula in different populations.

Harasymowycz et $a l^{7}$ suggested the RADAAR-1 formula using a larger disc-smaller disc comparison, based on the fact that RA increases to a smaller degree than CA with increases in the disc size. It was found that the RADAAR-1 measurements were significantly correlated with IOP and the degree of glaucomatous optic nerve damage in glaucoma patients. In this study, however, they did not test the ability of RADAAR-1 to discriminate between normal and glaucomatous eyes.

In the present study, the ranges of results with RADAAR using different formulae were determined for subjects in the different diagnostic categories (Figure 2). In general, $\mathrm{SD}$ of RADAAR mean values using different formulae was higher in the group with definitive glaucoma compared with the others groups signalling the great variation of RADAAR values in this diagnostic group. This variation of SD was higher with mean RADAAR-1values compared with mean RADAAR-2 and RADAAR-3 values. RADAAR-1 values correlated with
IOP and DDLS of the worse eye. The smaller the RADAAR-1 value, the greater was the IOP and DDLS stage of the 'worse eye', thus confirming the correlation of RADAAR-1 with the degree of severity of the disease. The absence of correlation of RADAAR-1 with the differences in IOP and DDLS, however, may be explained by the absence of a great asymmetrical glaucomatous damage in this study population. This is illustrated by both the lack of significant statistical difference in neuroretinal RA between the eyes in the group of definitive glaucoma (Table 3 ) and the finding of the existence of great correlation between inter-eye RA difference, and RADAAR-1 measurements in the same group of diagnosis. Also, this study included only subjects with diagnosis of primary open-angle glaucoma comparing with previous study populations, ${ }^{11}$ which included pseudoexfoliative glaucoma and low-tension glaucoma, which may exhibit more asymmetric damage.

Hawker et $a l,{ }^{8}$ in their first paper, chose to compare eyes not on a relative scale, but on an absolute one by subtracting smaller disc parameters from the larger disc parameters, arguing that by comparing the results between eyes by division rather than subtraction results in a loss of potentially useful information. In the current study, this formula (RADAAR-2) failed to discriminate between the different diagnostic groups as well as RADAAR-3, a formula used in a later paper. ${ }^{9}$ However, a statistical significant correlation found between RADAAR-2 measurement and IOP of the worse eye as well as inter-eye IOP difference, especially in the definitive glaucoma group, and also the correlation found between RADAAR-3 and the clinical CDAR of the worse eye, tends to confirm again the correlation of RADAAR value with the degree of severity of the disease.

The classification of different diagnostic groups is one of the key points on which all the results of this study depend. The ideal diagnosis correlates structural (optic nerve head) and functional (visual field) damage. This was done in our study but the optic nerve head exam was performed by one glaucoma specialist and documented using the DDLS. The DDLS has been shown to have an area under the curve of 0.95 for perimetric glaucoma. ${ }^{23}$ We used FDT perimetry as frequencydoubling technology, which has been reported to be a good predictor of future standard automated perimetry visual field defects. ${ }^{24-26}$ We considered two or more adjacent squares of relative loss as abnormal using the C-20-5. In a previous study, this definition was associated with a specificity of $85 \%$ and a sensitivity of $66.7 \%$ for glaucoma, ${ }^{12}$ and in the current study influenced the distribution of patients in their various diagnostic groups. Hawker et al ${ }^{9}$ chose to define glaucoma based on visual fields because of the problem of disagreement in 
classifying optic nerve heads clinically. ${ }^{27,28}$ Additionally, they took into account only two groups of diagnoses: normal and glaucoma. In this study, we chose to integrate glaucoma suspect groups, including possible and probable glaucoma diagnostic groups, as it reflects the clinical reality that all data must be taken into account when deciding the likelihood that a patient may have glaucoma.

Current study limitations include the accuracy of drawing the HRT contour line, as well as the fact that only one ophthalmologist examined subjects. The relatively small number of subjects in the different diagnostic groups, as well as the difference from where they were recruited (normals mostly from screening and glaucomas mostly in a clinic setting) also induces biases.

In conclusion, knowledge of RADAAR distribution in various populations of patients may aid in the clinical diagnosis of asymmetric glaucomatous damage. Despite its poor correlation with different clinical variables, the RADAAR-1 formula seems to best distinguish between different diagnostic groups. Further studies are needed to determine the validity of RADAAR in detecting asymmetric glaucoma damage in various populations as well as to describe the utility of RADAAR in detecting glaucoma in its various stages.

\section{Summary}

What was known before

- Rim area to disc area asymmetry ratio (RADAAR) was stated based on the fact that by comparing the two eyes of each subject, asymmetry takes into account interindividual measures variation.

What this study adds

- Knowledge of RADAAR distribution in various diagnostic groups may aid in clinical diagnosis of asymmetric glaucomatous damage.

\section{Conflict of interest}

The authors declare no conflict of interest.

\section{Acknowledgements}

This work was funded by Fonds de Recherche en Ophtalmologie de l'Université de Montréal (FROUM), Glaucoma Research Society of Canada (GRSC) and Foundation du glaucome du Québec.

\section{References}

1 Jonas JB, Grundler AE. Correlation between mean visual field loss and morphometric optic disk variables in the open-angle glaucomas. Am J Ophthalmol 1997; 124: 488-497.
2 Read RM, Spaeth GL. The practical clinical appraisal of the optic disc in glaucoma: the natural history of cup progression and some specific disc-field correlations. Trans Am Acad Ophthalmol Otolaryngol 1974; 78: OP255-OP274.

3 Sommer A, Katz J, Quigley HA, Miller NR, Robin AL, Richter RC et al. Clinically detectable nerve fiber atrophy precedes the onset of glaucomatous field loss. Arch Ophthalmol 1991; 109: 77-83.

4 Zeyen TG, Caprioli J. Progression of disc and field damage in early glaucoma. Arch Ophthalmol 1993; 111: 62-65.

5 Balazsi AG, Drance SM, Schulzer M, Douglas GR. Neuroretinal rim area in suspected glaucoma and early chronic open-angle glaucoma. Correlation with parameters of visual function. Arch Ophthalmol 1984; 102: 1011-1014.

6 Hitchings RA, Brown DB, Anderton SA. Glaucoma screening by means of an optic disc grid. Br J Ophthalmol 1983; 67: 352-355.

7 Harasymowycz P, Davis B, Xu G, Myers J, Bayer A, Spaeth GL. The use of RADAAR (ratio of rim area to disc area asymmetry) in detecting glaucoma and its severity. Can J Ophthalmol 2004; 39: 240-244.

8 Hawker MJ, Vernon SA, Ainsworth G, Hillman JG, MacNab HK, Dua HS. Asymmetry in optic disc morphometry as measured by Heidelberg retina tomography in a normal elderly population: the Bridlington Eye Assessment Project. Invest Ophthalmol Vis Sci 2005; 46: 4153-4158.

9 Hawker MJ, Vernon SA, Tattersall CL, Dua HS. Detecting glaucoma with RADAAR: the Bridlington Eye Assessment Project. Br J Ophthalmol 2006; 90: 744-748.

10 Harasymowycz PJ, Papamatheakis DG, Fansi AK, Gresset J, Lesk MR. Validity of screening for glaucomatous optic nerve damage using confocal scanning laser ophthalmoscopy (Heidelberg retina tomograph II) in high-risk populations: a pilot study. Ophthalmology 2005; 112: 2164-2171.

11 Bayer A, Harasymowycz P, Henderer JD, Steinmann WG, Spaeth GL. Validity of a new disk grading scale for estimating glaucomatous damage: correlation with visual field damage. Am J Ophthalmol 2002; 133: 758-763.

12 Gardiner SK, Anderson DR, Fingeret M, McSoley JJ, Johnson CA. Evaluation of decision rules for frequencydoubling technology screening tests. Optom Vis Sci 2006; 83: 432-437.

13 Patel SC, Friedman DS, Varadkar P, Robin AL. Algorithm for interpreting the results of frequency doubling perimetry. Am J Ophthalmol 2000; 129: 323-327.

14 Bartz-Schmidt KU, Weber J, Heimann K. Validity of two-dimensional data obtained with the Heidelberg retina tomograph as verified by direct measurements in normal optic nerve heads. Ger J Ophthalmol 1994; 3: 400-405.

15 Weinreb RN, Zangwill LM. Imaging technologies for assessing neuroprotection in glaucomatous optic neuropathy. Eur J Ophthalmol 1999; 9(Suppl 1): S40-S43.

16 Wollstein G, Garway-Heath DF, Hitchings RA. Identification of early glaucoma cases with the scanning laser ophthalmoscope. Ophthalmology 1998; 105: 1557-1563.

17 Schlamp CL, Li Y, Dietz JA, Janssen KT, Nickells RW. Progressive ganglion cell loss and optic nerve degeneration in DBA/2J mice is variable and asymmetric. BMC Neurosci 2006; 7: 66.

18 Van-der-Helm FGM. Hydrophthalmia and its treatment. A general study based on 630 cases in the Netherlands. Bibl Ophth 1963; 61: 1-64. 
19 Ong LS, Mitchell P, Healey PR, Cumming RG. Asymmetry in optic disc parameters: the Blue Mountains Eye Study. Invest Ophthalmol Vis Sci 1999; 40: 849-857.

20 Durukan AH, Yucel I, Akar Y, Bayraktar MZ. Assessment of optic nerve head topographic parameters with a confocal scanning laser ophthalmoscope. Clin Experiment Ophthalmol 2004; 32: 259-264.

21 Vernon SA, Hawker MJ, Ainsworth G, Hillman JG, Macnab HK, Dua HS. Laser scanning tomography of the optic nerve head in a normal elderly population: the Bridlington eye assessment project. Invest Ophthalmol Vis Sci 2005; 46: 2823-2828.

22 Nakamura H, Maeda T, Suzuki Y, Inoue Y. Scanning laser tomography to evaluate optic discs of normal eyes. Jpn J Ophthalmol 1999; 43: 410-414.

23 Danesh-Meyer HV, Gaskin BJ, Jayusundera T, Donaldson M, Gamble GD. Comparison of disc damage likelihood scale, cup to disc ratio, and Heidelberg retina tomograph in the diagnosis of glaucoma. Br J Ophthalmol 2006; 90: 437-441.
24 Kondo Y, Yamamoto T, Sato Y, Matsubara M, Kitazawa Y. A frequency-doubling perimetric study in normal-tension glaucoma with hemifield defect. J Glaucoma 1998; 7: 261-265.

25 Bayer AU, Erb C. Short wavelength automated perimetry, frequency doubling technology perimetry, and pattern electroretinography for prediction of progressive glaucomatous standard visual field defects. Ophthalmology 2002; 109: 1009-1017.

26 Medeiros FA, Sample PA, Weinreb RN. Frequency doubling technology perimetry abnormalities as predictors of glaucomatous visual field loss. Am J Ophthalmol 2004; 137: 863-871.

27 Varma R, Steinmann WC, Scott IU. Expert agreement in evaluating the optic disc for glaucoma. Ophthalmology 1992; 99: 215-221.

28 Azuara-Blanco A, Katz LJ, Spaeth GL, Vernon SA, Spencer F, Lanzl IM. Clinical agreement among glaucoma experts in the detection of glaucomatous changes of the optic disk using simultaneous stereoscopic photographs. Am J Ophthalmol 2003; 136: 949-950. 